\title{
Insights into the Action Mechanisms of Traditional Chinese Medicine in Osteoarthritis
}

\author{
Linfu Li, ${ }^{1}$ Haiqing Liu, ${ }^{1}$ Weimei Shi, ${ }^{1}$ Hai Liu, ${ }^{1}$ Jianqiong Yang, ${ }^{2}$ Daohua $\mathrm{Xu},{ }^{3}$ \\ Hao Huang, ${ }^{1}$ and Longhuo $\mathrm{Wu}^{1}$ \\ ${ }^{1}$ College of Pharmacy, Gannan Medical University, Ganzhou 341000, China \\ ${ }^{2}$ Department of Clinical Research Center, The First Affiliated Hospital of Gannan Medical University, Ganzhou 341000, China \\ ${ }^{3}$ Department of Pharmacology, Guangdong Medical University, Dongguan 523808, China
}

Correspondence should be addressed to Hao Huang; hwaohuanghao@gmail.com and Longhuo Wu; longhwu@hotmail.com

Received 16 September 2016; Revised 27 November 2016; Accepted 14 December 2016; Published 22 January 2017

Academic Editor: Isabel Andújar

Copyright (C) 2017 Linfu Li et al. This is an open access article distributed under the Creative Commons Attribution License, which permits unrestricted use, distribution, and reproduction in any medium, provided the original work is properly cited.

Osteoarthritis (OA) is a chronic degenerative joint disease characterized by articular cartilage destruction, synovial inflammation, and osteophyte formation. No effective treatments are available. The current pharmacological medications such as nonsteroidal anti-inflammatory drugs (NSAIDs) and analgesics, accompanied by possible adverse effects, might ameliorate OA symptoms. But they do not arrest the progression of OA. Traditional Chinese medicine (TCM) provides medical value by modification of disease and symptoms in OA. Valuable work on exploring TCM merits for OA patients has been investigated using modern technologies, although the complicated interacting network among the numerous components indicates the uncertainty of target specification. This review will provide an overview of the action mechanism of TCM in the last 5 years, discussing the TCM activities of antiinflammation, antiapoptosis, antioxidation, anticatabolism, and proliferation in OA. TCM is a proposed medical option for OA treatment.

\section{Introduction}

Osteoarthritis (OA) is a deteriorating joint disease and becomes the major cause of chronic disability, especially for the aged people. The pathological developments in OA include the destruction of cartilage, the inflammation of synovium, the formation of osteophyte, the thickening of subchondral bone, and the degeneration of chondrocytes [1]. The etiological risk for OA involves various factors, including inflammation, oxidative stress, mechanical stress, join injury, age, and other systemic diseases [2]. To date, there is no effective treatment for OA. Unfortunately, the aging society will produce more OA patients. More than 27 million Americans with over $\$ 185.5$ billion annual medical expenditures have posed a great significant economic burden in USA [3].

The balance driving for anabolic and catabolic activities in articular cartilage is controlled by signaling pathways. However, this balance is tilted towards joint destruction.
Various signaling pathways are involved in OA to impair the homeostasis of articular cartilage. These include $\mathrm{Wnt} / \beta$ catenin pathway [4], the nuclear factor- $\kappa \mathrm{B}(\mathrm{NF}-\kappa \mathrm{B})$ pathway [5], the p38, ERK1/2 and JNK MAP kinase [6], and the PI-3 kinase- (PI3K-) Akt pathway [6], which have been comprehensively reviewed $[7,8]$. Although the inflammation of synovial membrane and the degeneration of articular cartilage are the two major features of $\mathrm{OA}$, the underlying mechanisms responsible for joint destruction are still unclear. With Lack of detailed and clear information about the pathology of OA, it is quite difficult to develop effective strategies for OA management.

Currently, the goals for managing $\mathrm{OA}$ are to ameliorate the painful symptoms, minimize disability, and enhance the life quality. The treatment options recommended in clinic are involving surgery, the existing pharmacological medication, or nonpharmacological therapies, such as exercises and weigh loss [9]. The pharmacological intervention in clinical practice mainly consists of nonsteroidal anti-inflammatory drugs 


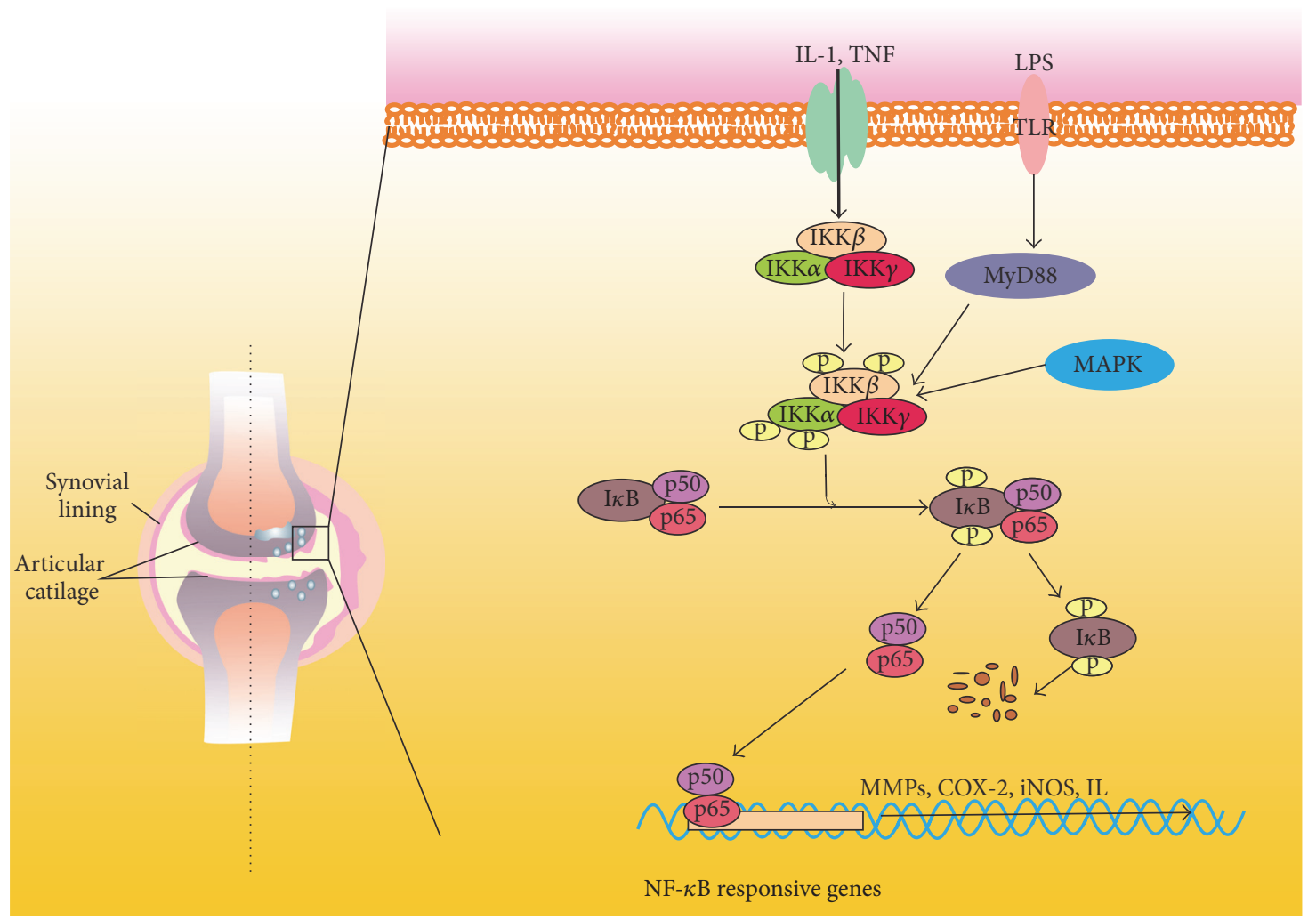

FIGURE 1: The activity of NF- $\kappa \mathrm{B}$ signaling in OA chondrocytes. NF- $\kappa \mathrm{B}$ signaling may be activated by many detrimental stimuli, such as IL-1, TNF, and LPS, leading to upregulation of MMPs, COX-2, iNOS, and IL. Crosstalk with MAPK also promotes NF- $\kappa$ B signaling.

(NSAIDs), analgesics, hyaluronic acid, and corticosteroids [10]. However, these pharmacological medications do not meet patients' expectancy and even cause serious side effects. Traditional medicine including some nutraceuticals acts as the promising alternative medicine as a treatment option for $\mathrm{OA}$ and might be easily adopted by clinicians and patients (Table 1). Evidences that support physiological and functional value of nutraceuticals for $\mathrm{OA}$ have been comprehensively reviewed by Henrotin et al. [11] and Leong et al. [12]. In this review, we will summarize the action features of TCM and their components that positively contribute to ameliorate the pathological changes in cartilage and chondrocytes.

\section{Anti-Inflammatory Activity of TCM}

Inflammation is closely related not only to symptoms and signs but also to progressive cartilage loss in OA. The lowgrade inflammatory response greatly triggers the development of OA. IL- $1 \beta$ and TNF $\alpha$ are the two major catabolic inflammatory cytokines to drive the degradation of cartilage. The proinflammatory cytokines implicated in OA can activate $\mathrm{NF}-\kappa \mathrm{B}$ signaling (Figure 1), which in turn controls the expression of these cytokines. This makes a vicious cycle [13]. In addition, $\mathrm{NF}-\kappa \mathrm{B}$ signaling interacts with other pathways to deteriorate the degeneration of cartilage.

Inflammatory cytokines induce upregulation of the catabolic activity of matrix metalloproteinases (MMPs) through activation of NF- $\kappa \mathrm{B}$ signaling pathway. Aucubin, a natural occurring compound from Aucuba japonica and Eucommia ulmoides, has been reported to exhibit antiinflammatory activity by inhibiting the degradation and phosphorylation of $\mathrm{I} \kappa \mathrm{B}$ and blocking the translocation of NF$\kappa \mathrm{B}$ p65. In IL- $1 \beta$-stimulated chondrocytes, aucubin downregulates the increased expression of MMPs, COX-2, and iNOS and the enhanced production of NO [14]. Honokiol, extracted from Magnolia officinalis, possesses anti-inflammatory and antioxidative activities. Honokiol can significantly inhibit IL$1 \beta$-induced upregulation of IKK/I $\kappa \mathrm{B} \alpha / \mathrm{NF}-\kappa \mathrm{B}$ signaling pathway, leading to suppression of the expression of iNOS, COX2, NO, PGE 2 , IL-6, and MMP-13 [15]. However, honokiol administration might induce embryo-fetal development toxicity [16]. Heat shock protein $90 \beta$ (Hsp90 $\beta$ ), a member of Hsp90 family of protein chaperones, has been reported to be related to inflammation in rat arthritis. Celastrol is known to suppress immune activation. Celastrol is also an inhibitor of Hsp90 $\beta$ to significantly downregulate the expression of MMP-1, MMP-3, MMP-13, iNOS-2, and COX-2 induced by IL- $1 \beta$ [17].

Black pepper (Piper nigrum), a spice in general diets, is often used as a traditional medicine. Piperine is an active phenolic constituent and has antiarthritis and anti-inflammatory effects on IL- $1 \beta$-induced fibroblast-like synoviocytes to benefit some inflammatory diseases that are accompanied by pain, such as OA and RA. Reports show that piperine significantly inhibits IL- $1 \beta$-induced activation of NF- $\kappa$ B signaling, leading to downregulation of COX-2, PGE2, iNOS, NO, and 


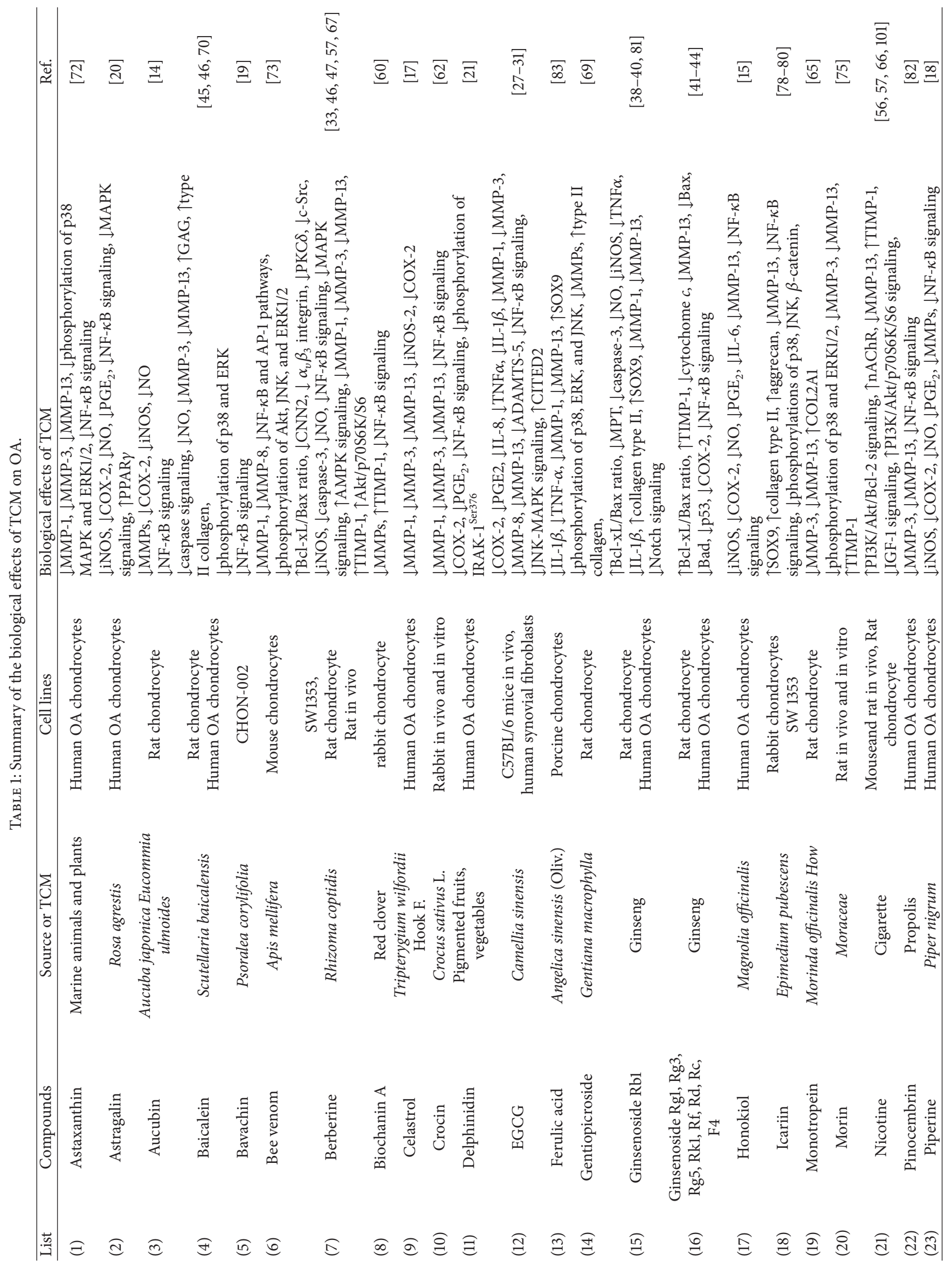




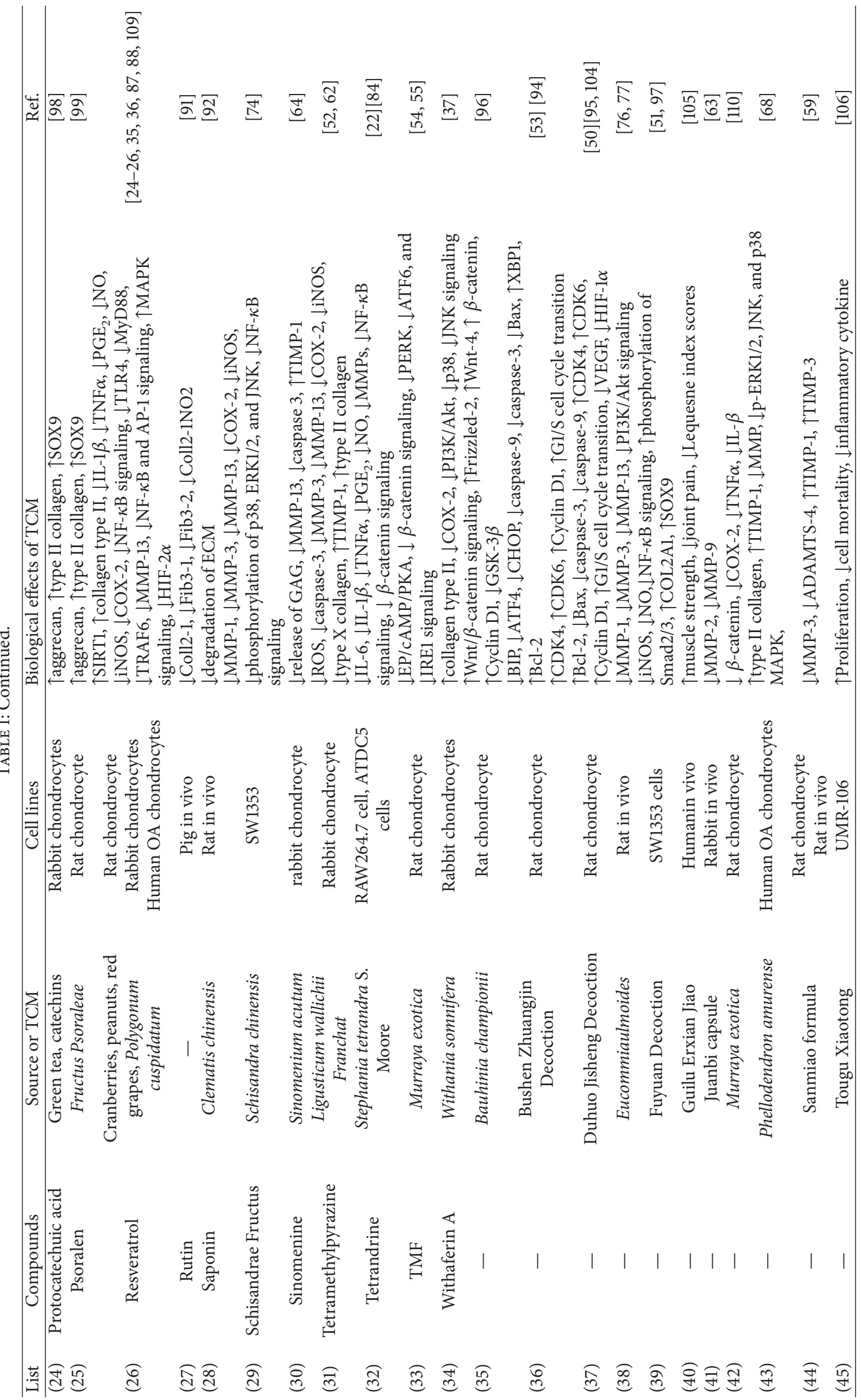


MMPs in OA [18]. Bavachin, isolated from Psoralea corylifolia, is one of medicinal phytoestrogens that exhibit antiinflammatory activity. Bavachin potently protects cartilage from inflammation-mediated damage through decreasing IL- $1 \beta$-induced nuclear translocation of p 65 and p50 and degradation of $\mathrm{I} \kappa \mathrm{B} \alpha$ but not activator protein-1 (AP-1) DNAbinding activity [19]. Astragalin, a main constituent isolated from Rosa agrestis, exhibits anti-inflammatory effects in OA by activating PPAR $\gamma$, leading to inhibition of IL- $1 \beta$-induced $\mathrm{NF}-\kappa \mathrm{B}$ and MAPK activation and reduction of $\mathrm{NO}$ and $\mathrm{PGE}_{2}$ production, as well as iNOS and COX-2 expression [20]. Delphinidin is an anthocyanidin compound isolated from pigmented fruits and vegetables and possesses antioxidative and anti-inflammatory activities. It is found that delphinidin can inhibit IL- $1 \beta$-induced NF- $\kappa$ B signaling through modulating the phosphorylation of IRAK- ${ }^{\text {Ser376 }}$, resulting in suppression of COX-2/ $\mathrm{PGE}_{2}$ expression in OA chondrocytes [21]. Tetrandrine, a main bisbenzylisoquinoline alkaloid extracted from Stephania tetrandra S. Moore, inhibits the expression of IL6 , IL- $1 \beta, \mathrm{TNF} \alpha, \mathrm{PGE}_{2}$, and NO via blocking $\mathrm{I} \kappa \mathrm{B} \alpha$ and NF$\kappa \mathrm{B}$ p65 phosphorylation in LPS-induced ATDC5 cells [22]. Possibly, tetrandrine administration can cause pulmonary toxicity and metabolic disorder [23].

Resveratrol (trans-3,4-trihydroxystilbene), a natural phytoalexin isolated from cranberries, peanuts, the skin of red grapes, and the root of the weed Polygonum cuspidatum, possesses anti-inflammatory, antitumor, and antioxidative activities. Resveratrol is a natural SIRT1 activator to exhibit an anti-inflammatory activity via inhibition of NF- $\kappa$ B. The mechanism might be that resveratrol-activated SIRT1 suppresses not only the nuclear translocation of NF- $\kappa$ B but also the acetylation of p65, leading to downregulation of iNOS expression [24]. Resveratrol has been confirmed to inhibit the expression of COX-2 and iNOS, IL- $1 \beta$, and $\mathrm{TNF} \alpha$ via downregulation of NF- $\kappa \mathrm{B}$ signaling pathway [25]. It also increases the production of type II collagen at mRNA and protein levels in the superficial and middle zones, but much less in the deep zone in cartilage [26].

Catechins, isolated from Camellia sinensis, are the main effective compounds of tea polyphenols. (-)Epigallocatechin-3-gallate (EGCG), one of the most abundant catechins, is the great nutraceutical tea polyphenol in OA, which has been reviewed by Ahmed [27]. EGCG can significantly suppress the expression of COX-2, PGE2, and IL-8 dose-dependently in IL- $1 \beta$-induced human synovial fibroblasts via reducing the phosphorylation of IKK $\beta$ [28]. Calcium crystals formation is considered to be a factor implicated in synovial inflammation. Studies show that EGCG exhibits chondroprotective activity through reducing the inflammatory response induced by calcium pyrophosphate crystals in vitro [29]. EGCG might be the potential candidate for $\mathrm{OA}$ treatment by targeting epithelial neutrophil activating peptide-78 (ENA78 ), granulocyte macrophage colony stimulation factor (GM-CSF), growth-related oncogene (GRO), GRO- $\alpha$, IL-6, IL-8, monocyte chemotactic protein-1 (MCP-1), MCP-3, macrophage inflammatory protein-1beta (MIP-1 $\beta$ ), granulocyte chemotactic protein-2 (GCP-2), MIP-3alpha, interferon-gamma-inducible protein-10 (IP-10), nucleosome assembly protein-2 (NAP-2), and leukemia inhibitory factor (LIF) through downregulation of NF- $\kappa$ B and JNKMAPK signaling pathways [30]. In the EGCG-treated (intraperitoneal injection) mice, articular cartilage shows downregulation of MMP-1, MMP-3, MMP-8, MMP-13, ADAMTS-5, IL- $1 \beta$, and TNF $\alpha$ mRNA and upregulation of $\mathrm{CBP} / \mathrm{p} 300$ interacting transactivator with ED-rich tail 2 (CITED2), which suppresses MMPs transcription [31].

Connective tissue growth factor (CTGF; also known as CCN2), an inflammatory cytokine, is highly expressed in OA. CCN2 can increase the production of IL- $1 \beta$ in osteoarthritis synovial fibroblasts through generation of $\alpha_{v} \beta_{3} / \alpha_{v} \beta_{5}$ integrin-dependent ROS and subsequent activation of signalregulating kinase 1 (ASK1), p38/JNK, and NF- $\kappa$ B signaling pathways. Berberine, an isoquinoline alkaloid isolated from the Rhizoma coptidis, effectively prevents cartilage degradation by antagonizing the effect of CNN2 [32]. In addition, it has been demonstrated that downregulation of $\alpha_{v} \beta_{3}$ integrin is also modulated by berberine through posing the inhibitory effect on $\mathrm{PKC} \delta$, $c-\mathrm{Src}$, and NF- $\kappa \mathrm{B}$ signaling pathways in human chondrosarcoma [33]. Recently, berberine at a relative dose can stimulate the hermetic dose response from clinical applications, particularly in the management of cancer [34]. Toll-like receptor 4 (TLR4) elicits inflammatory responses to develop antigen-specific adaptive immune responses. TLR4 is not only expressed by immune cells, but also expressed by nonprofessional antigen presenting cells (such as cartilage chondrocytes). It has been demonstrated that the expression of TLR4 is increased in chondrocytes from OA patients, indicating that TLR4 might be involved in the OA development [35]. Study shows that resveratrol significantly suppresses the activation of NF- $\kappa \mathrm{B}$ signaling induced by TLR 4 in RAW264.7 cells [36]. In human chondrocytes, resveratrol can remarkably suppress the upregulation of TLR4 and the downstream target MyD88 and TRAF6 induced by IL-1 $\beta$ [37].

On the other hand, Withaferin A, isolated from Withania somnifera, can induce the loss of type II collagen, reactive oxygen species, and inflammation in rabbit articular chondrocytes. Evidences show that Withaferin A stimulates downregulation of type II collagen and upregulation of COX2 through activation of PI3K/Akt, p38, and JNK signaling pathways [38].

\section{Antiapoptosis and Antioxidative Activities of TCM}

Chondrocyte apoptosis is closely related to the progression of OA. Chondrocytes apoptosis can be stimulated by reactive oxygen species (ROS), which can be produced by hydrogen peroxide $\left(\mathrm{H}_{2} \mathrm{O}_{2}\right)$. Proinflammatory cytokines can enhance the level of ROS via activation of NF- $\kappa$ B signaling pathway. ROS promotes the mitochondrial permeability transition (MPT), resulting in release of cytochome $c$ into the cytoplasm. Ginsenoside Rbl, a pivotal component of ginseng, exhibits the activity of inhibiting $\mathrm{H}_{2} \mathrm{O}_{2}$-induced MPT, the expression of caspase- 3 , and the imbalance of $\mathrm{Bcl}-\mathrm{xL} / \mathrm{Bax}$ ratio [39]. $\mathrm{Rbl}$ inhibits $\mathrm{H}_{2} \mathrm{O}_{2}$-elicited $\mathrm{NO}$ and iNOS production and suppresses the proinflammatory cytokines IL- $1 \beta$ and TNF $\alpha$ expression [40, 41]. Rg1, Rg3, Rg5, Rk1, Rf, Rd, Rc, and 
F4 are saponins and have similar chondroprotective activity [42-44]. They inhibit IL-1 $\beta$-induced chondrocytes apoptosis by enhancing $\mathrm{Bcl}-2 / \mathrm{Bax}$ ratio and inhibiting the cytochome $c$ release. Also, they promote the expression of TIMP-1 and inhibit the expression of MMP-13 through downregulating the PI3K/Akt signaling pathway [42]. Bax, Bad, p53, COX2 , and p65 are closely related to chondrocytes apoptosis. Ro, another oleanolic acid-type ginsenoside, suppresses cell apoptosis by inhibiting the levels of Bax and Bad, decreasing phosphorylation of $\mathrm{p} 53$, and promoting the expression of Bcl$\mathrm{xL}$ and PCNA. Moreover, Ro inhibits the phosphorylation of NF- $\kappa$ B p 65 induced by IL-1 $\beta$ [45].

Proinflammatory cytokines such as IL-1 $\beta$ and TNF$\alpha$ (IT) can stimulate the caspase signaling to induce cell apoptosis. Baicalein, a main active compound isolated from Scutellaria baicalensis Georgi, exhibits antiapoptotic activity by decreasing $\mathrm{NO}$ production and inhibiting the caspase cascade activation [46]. NO donor sodium nitroprusside (SNP) is commonly used as an inducer to trigger apoptosis. Berberine has been reported to reverse SNP-induced cytoskeletal remodeling and chondrocytes apoptosis with downregulation of the expression of iNOS and caspase-3 and upregulation of $\mathrm{Bcl} / \mathrm{Bax}$ ratio and production of type II collage. These are accompanied by activation of AMPK phosphorylation and suppression of p38 MAPK phosphorylation [47]. Because of poor aqueous solubility, berberine has a low bioavailability and short biological half-life. Berberineloaded chitosan nanoparticles are designed. They confer stronger activity of antiapoptosis in OA through qRT-PCR, Western blot, and immunohistochemical analyses of caspase3, Bcl-2, and Bax expression [48].

NO and ROS are responsible for SNP-induced chondrocytes apoptosis through an intrinsic apoptosis pathway. Resveratrol has been demonstrated to scavenge SNPinduced ROS, instead of NO, to remarkably prevent chondrocytes apoptosis [49]. Using atomic force microscopy (AFM), resveratrol potently prevent SNP-induced chondrocytes changes, which include shrunk, round, lamellipodia contraction, and aggregation of the cytoskeleton, decrease in adherent junctions among cells, and decrease in the expression of cytoskeletal proteins [50]. Duhuo Jisheng decoction, a Chinese traditional herbal formula, increases Bcl-2 expression, whereas it decreases the expression of Bax, caspase3 , and caspase- 9 induced by SNP to inhibit chondrocytes apoptosis in a mitochondrial dependent manner [51]. Fuyuan Decoction (FYD) has been demonstrated to decrease $\mathrm{I} \kappa \mathrm{B} \alpha$ degradation and reduce the content of p65 in the nucleus induced by IL-1 $\beta$, resulting in suppression of iNOS and NO expression in SW1353 cells [52]. Tetramethylpyrazine, a main active component isolated from Ligusticum wallichii Franchat, inhibits the chondrocytes apoptosis through downregulation of ROS and caspase-3 expression and maintenance of mitochondrial membrane potential [53].

Chondrocytes apoptosis can also be triggered by endoplasmic reticulum (ER) stress, which is caused by the accumulation of unfolded or misfolded proteins. In tunicamycin(TM-) induced chondrocytes, Bushen Zhuangjin Decoction decreases the mRNA and protein expression of BIP, ATF4, CHOP, caspase-9, caspase- 3 , and Bax and increases the expression of XBP1 and $\mathrm{Bcl}-2$ [54]. Our research group shows that $5,7,3^{\prime}, 4^{\prime}$-tetramethoxyflavone (TMF), an active compound isolated from Murraya exotica (L.), can inhibit chondrocytes apoptosis through downregulation of the increased $\mathrm{EP} / \mathrm{cAMP} / \mathrm{PKA}$ signaling pathway and $\beta$-catenin signaling pathway induced by $\mathrm{PGE}_{2}$ [55]. In addition, TMF also suppresses TM-induced ER stress by downregulating the three membrane proteins PERK, ATF6, and IRE1 signaling pathways [56]. Nicotine activates the nicotinic acetylcholine receptors (nAChR) to resist cell apoptosis and promote cell proliferation [57]. It has been demonstrated that nicotine blocks cell apoptosis by neutralizing IL- $1 \beta$-induced downregulation of PI3K/Akt signaling pathway, including $\mathrm{PI} 3 \mathrm{~K} / \mathrm{Akt} / \mathrm{Bcl}-2$ signaling in chondrocytes [58].

\section{Anticatabolic Activity of TCM}

Articular cartilage extracellular matrix (ECM) plays a crucial role in regulating chondrocyte metabolism and functions. ECM is constituted primarily by type II collagen and large networks of proteoglycans (PGs) that contain glycosaminoglycan (GAG), hyaluronic acid (HA), and chondroitin sulfate (CS) [59]. Destruction of the articular cartilage in OA might be due to the combination of increased degradation of ECM, decreased production of ECM, and chondrocyte death. MMPs and a disintegrin and metalloproteinase with thrombospondin motifs (ADAMTS) play the pivotal roles in the overactive catabolic destruction of cartilage.

Sanmiao formula, a Chinese traditional medicinal prescription since the Ming Dynasty, exhibits protective activity for cartilage through decreasing the expression of MMP-3 and ADAMTS- 4 and augmenting the expression of TIMP1 and TIMP-3. In addition, it also inhibits the expression of IL- $1 \beta$ and TNF $\alpha$, which are associated with activation of NF$\kappa \mathrm{B}$ signaling regulating the activity of MMPs [60]. Biochanin $\mathrm{A}$, an isoflavone isolated from red clover, exhibits antiallergic, anticancer, and anti-inflammatory activity. Investigation indicates that biochanin A inhibits the increased expression of MMPs and enhances the decreased TIMP-1 expression induced by IL-1 $\beta$ in chondrocytes, which might be associated with attenuation of NF- $\kappa$ B signaling [61]. Crocin is a main active compound isolated from Crocus sativus L. (saffron) and does not harm live and other organ's function within the pharmacological doses [62]. It has been showed that Crocin ameliorates cartilage degeneration and decreases the expression of MMP-1, MMP-3, and MMP-13 through inhibition of $\mathrm{NF}-\kappa \mathrm{B}$ signaling [63]. Juanbi capsule is a Chinese medicine for preventing OA. It has been found that Juanbi capsule in vivo can effectively protect cartilage and significantly decrease serum MMP-2 and MMP-9 levels [64]. Sinomenine, an alkaloid isolated from Sinomenium acutum, can dosedependently inhibit the release of glycosaminoglycans (GAG) and the expression of MMP-13 and caspase 3 and enhance the activity of TIMP-1, leading to prevention of DNA fragment and cell apoptosis in chondrocytes [65]. Monotropein, an iridoids glycoside isolated from the roots of Morinda officinalis How, inhibits IL-1 $\beta$-induced upregulation of MMP3 and MMP-13 and promotes the expression of COL2A1 [66]. 
Nicotine promotes protein synthesis and modulates the activity of MMP-13 and TIMP-1 to maintain ECM balance in cartilage through upregulating PI3K/Akt/p70S6K/S6 signaling pathway [67]. In an experimental rat OA model, berberine decreases the expression of MMP-1, MMP-3, and MMP-13 whereas it increases TIMP-1 at the mRNA and protein levels [68]. The underlying mechanism might be associated with activation of Akt and p70S6K/S6 signaling pathways, which are involved in the chondroprotective activity of berberine in maintenance of cell survival and promotion of matrix production [58]. Phellodendron amurense is widely used as an anti-inflammatory and immunostimulatory medicine. Evidence shows that Phellodendron amurense protects joint cartilage from induction of IL- $1 \alpha$ through inhibiting the release of proteoglycan and the degradation of type II collagen, decreasing the activities of aggrecanases, MMP, p-ERK1/2, JNK, and p38 MAPK signaling, and increasing the activity of TIMP-1 [69]. Gentiopicroside, a main effective component of secoiridoid glycosides isolated from Gentiana macrophylla Pall, inhibits the phosphorylation of p38, ERK, and JNK and the expression of MMPs induced by IL-1 $\beta$. In addition, it enhances the production of type II collagen [70]. Baicalein reduces the expression of MMP-3 and MMP-13 and enhances the production of GAG and type II collagen [46]. These might be associated with downregulation of phosphorylation of p38 and ERK but not of JNK [71]. Tetramethylpyrazine has been showed to decrease the degradation of GAG and the expression of MMP-3, MMP-13, COX-2, iNOS, and type X collagen and increase the expression of TIMP-1 and type II collagen $[53,72]$.

Astaxanthin is a natural red carotenoid pigment and possesses antioxidative and anti-inflammatory activity in cartilage. Investigation indicates that astaxanthin can decrease the expression of MMP-1, MMP-3, and MMP-13, inhibit the phosphorylation of p38 MAPK and ERK1/2, and block the degradation of $\mathrm{I} \kappa \mathrm{B} \alpha$ in IL- $1 \beta$-induced chondrocytes [73]. Bee venom is a natural ingredient produced by the honey bee (Apis mellifera). It has been demonstrated that bee venom can inhibit the TNF $\alpha$-induced increased expression of MMP-1 and MMP- 8 through downregulation of NF- $\kappa$ B and AP-1 signaling pathways. In addition, bee venom also can suppress the phosphorylation of Akt, JNK, and ERK1/2 induced by TNF $\alpha$. But it does not affect p38 phosphorylation [74]. Schisandrae Fructus (SF) is the dried fruit of Schisandra chinensis (Turcz.) Baill. (Magnoliaceae). The ethanol extract of SF can significantly exhibit chondroprotective features and attenuate the expression of MMP-1, MMP-3, MMP-13, COX-2, and iNOS through suppression of NF$\kappa \mathrm{B}$ signaling and $\mathrm{p} 38, \mathrm{ERK} 1 / 2$, and JNK phosphorylation [75]. Morin (3,5,7,2', $4^{\prime}$-pentahydroxyflavone), a flavonoid extracted from the Moraceae family, possesses antioxidative, anti-inflammatory, and antitumor activities. Studies show that morin inhibits IL- $1 \beta$-induced phosphorylation of p38 and ERK1/2, decreases the expression of MMP-3 and MMP13, and increases TIMP-1 expression [76]. Investigation on the effect of an aqueous extract of Eucommia ulmoides on the articular cartilage has been carried out. The results show that a lower Mankin's grade induced by Eucommia is involved in histopathological examination. The levels of MMP-1, MMP-3, and MMP-13 in the serum and synovial fluid are negatively controlled by Eucommia [77]. These might be associated with downregulation of PI3K/Akt signaling pathway [78].

Icariin, isolated from Epimedium pubescens, is a pivotal effective compound to be related to multitherapeutic activities. Icariin exhibits chondroprotective effect and promotes the synthesis of ECM through upregulation of SOX9, type II collagen, and aggrecan in chondrocytes [79], which might be related to inhibition of $\mathrm{NF}-\kappa \mathrm{B}$ signaling, leading to the suppression of the increased expression of MMP-13 induced by IL- $1 \beta$ in vivo and in vitro [80]. MAPK and $\mathrm{Wnt} / \beta$ catenin signaling are also involved in this modulation. Icariin is positively related to the decreased phosphorylations of $\mathrm{p} 38, \mathrm{JNK}$, and $\beta$-catenin. This suggests that icariin exerts a promising chondrogenic effect on cartilage tissue engineering [81]. Rb1 upregulates the expression of chondrogenic genes type II collagen and SOX9. Whereas it downregulates the ECM catabolic factors MMP-1 and MMP-13 [40, 41]. Additionally, MMP-13 is positively associated with Notch signaling, which can be suppressed by Rb1 [82]. Pinocembrin, a flavonoid extracted from propolis, inhibits the nuclear translocation of $\mathrm{p} 65$ and phosphorylation and degradation of $\mathrm{I} \kappa \mathrm{B} \alpha$ induced by $\mathrm{TNF} \alpha$ in human chondrocytes, leading to suppression of MMP-3 and MMP-13 expression [83]. Ferulic acid is a natural occurring product from Angelica sinensis (Oliv.) Diels. Biological activity investigation shows that ferulic acid significantly downregulates the hydrogen peroxideinduced IL- $1 \beta$, TNF- $\alpha$, MMP-1, and MMP-13 expression and upregulates SOX9 gene expression [84]. Tetrandrine exhibits chondroprotective activity in vivo and in vitro through inhibiting the increased expression of $\beta$-catenin signaling (Figure 2) and MMPs induced by IL- $1 \beta$ and enhancing the expression of TMP-1 [85]. Qi-Fang-Xi-Bi-Granules (QFXBG) is TCM granules used for the treatment of OA. Fangchinoline and tetrandrine are used as the markers for quality control of QFXBG by HPLC in commercial [86].

In OA, advanced glycation end products (AGEs) are upregulated in cartilage. The accumulation of AGEs can decrease synthesis of proteoglycan and collagen and increase expression of MMPs. These are related to activation of NF- $\kappa \mathrm{B}$ and AP-1 signaling pathways [87]. It has been demonstrated that resveratrol can protect cartilage and suppress AGEsinduced expression of COX-2, iNOS, and MMP-13 through downregulating IKK-I $\kappa \mathrm{B} \alpha-\mathrm{NF}-\kappa \mathrm{B}$ and JNK/ERK-AP-1 signaling pathways [88]. Resveratrol can synergize curcumin to stimulate the MAPK signaling pathway in human chondrocytes in vitro [89]. Curcumin, a highly pleiotropic molecule extracted from the rhizomes of Curcuma longa, presents great potential and excellent safety profile for treating OA, although it has a low solubility and poor bioavailability. The nutraceutical effects of curcumin in OA development have been comprehensively reviewed [90], and here we are not ready to discuss it too much.

From the conventional wisdom of converting skin/hide matrix into leather, it has been hypothesized that polyphenols might cross-link with type II collagen through hydrophobic association and hydrogen bonding. Investigations indicate that polyphenols (combination of EGCG, quercetin, catechin, and tannic acid) administrated by intra-articular injection 


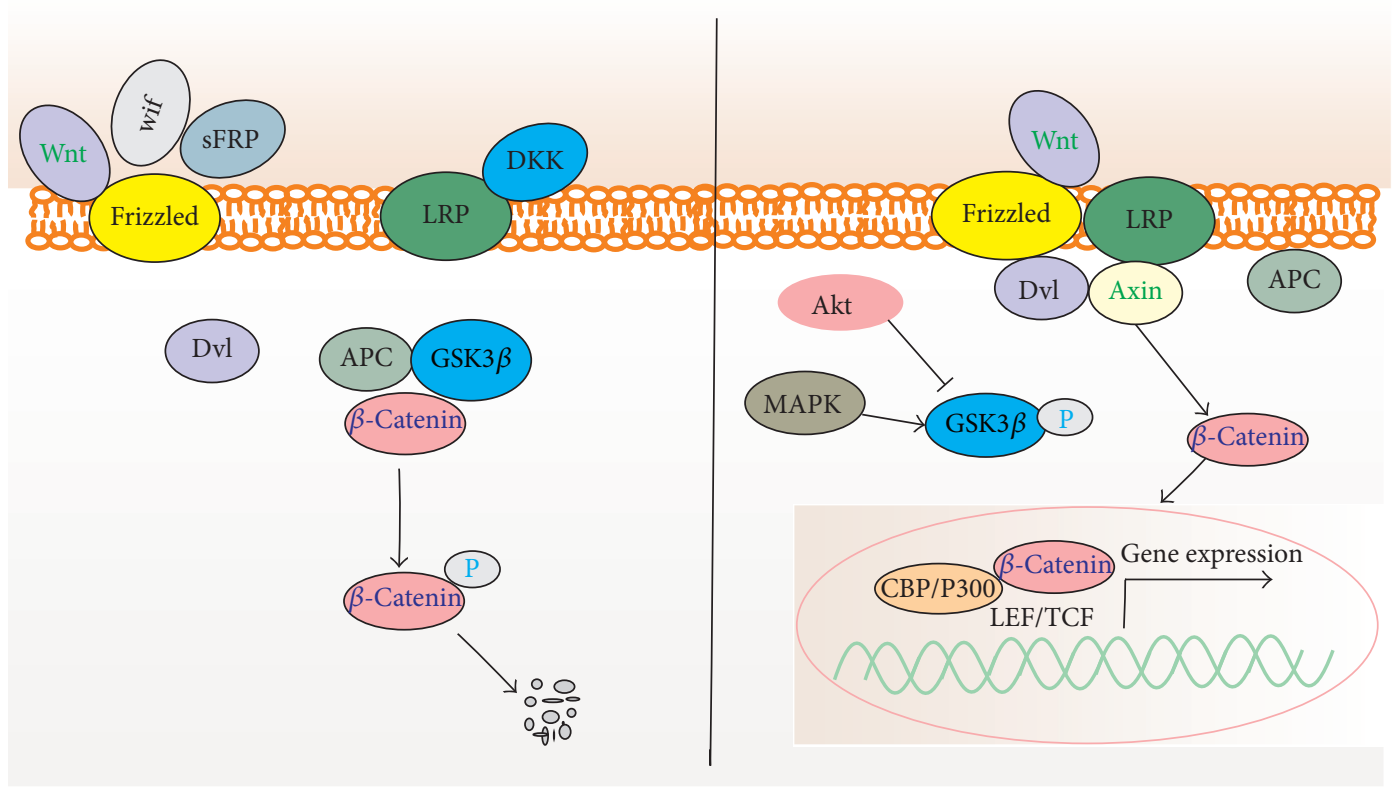

FIGURE 2: The Wnt/ $\beta$-catenin signaling in OA chondrocytes. Inhibitory phosphorylation of GSK-3 $\beta$ promotes the stability of $\beta$-catenin, which enters the nucleus for transcription.

can bind to collagen in bovine articular cartilage explants, which results in stabilization of cartilage collagen and resistance to degradation by collagenases [91]. To study the effects of polyphenols on anti-inflammation and anticatabolism in $\mathrm{OA}$, Horcajada et al. found that rutin and the mixture of rutin/curcumin could downregulate the expression of Coll21, the combination of rutin/curcumin could decrease Fib3-1 and Fib3-2 expression, and Coll2-1NO2 could be significantly downregulated by rutin, curcumin, and oleuropein [92].

The effect of the saponin fraction from Clematis chinensis Osbeck roots (SFC) on cartilage in a rat model induced by intra-articular injection of monosodium iodoacetate (MIA) has been investigated. The results show that SFC effectively ameliorates joint destruction and cartilage damage induced by MIA or SNP through blocking the degradation of ECM and preventing chondrocytes injury [93].

\section{Proliferative Activity of TCM}

Cyclin D1, CDK4, and CDK6 are the factors which interact with each other and form a complex to promote the cell cycle. Achyranthes bidentata polysaccharides are found in chondrocytes in vitro to induce G1/S cell cycle transition and type II collagen expression by upregulating the expression of CDK4, CDK6, and Cyclin D1, which are related to the Wnt/ $\beta$ catenin signaling pathway [94]. Bushen Zhuangjin Decoction [95] and Duhuo Jisheng Decoction [96] are also found to increase the expression of CDK4, CDK6, and Cyclin D1 and accelerate G1/S cell cycle transition, whereas they decrease the expression of p21. Bauhinia championi (Benth.) Benth. polysaccharides (BCBPs) also have been found to promote G1/S cell cycle transition and induce chondrocytes proliferation. BCBPs are composed of at least seven monosaccharides, such as rhamnose, D-(+) glucuronic acid, D-mannose, D- $(+)$ galacturonic acid, galactose, $\mathrm{D}$-glucose, and arabinose. It has been demonstrated that BCBPs can activate the expression of Wnt $/ \beta$-catenin signaling pathway (Figure 2 ) via upregulating the mRNA and protein expression of Frizzled-2, Wnt-4, $\beta$ catenin, and Cyclin D1 and downregulating the expression of GSK-3 $\beta$ [97].

TGF $\beta$ and SOX9 signaling pathways are of importance for the pathophysiology of joint cartilage. Generally, TGF $\beta$ predominantly phosphorylates Smad2/3 through ALK5, leading to forming a complex with Smad4 and translocating into the nucleus to regulate the target genes expression such as aggrecan and type II collagen. Fuyuan Decoction, a Chinese traditional herbal formula, can promote chondrocytes proliferation and reverse the decreased phosphorylation of Smad2/3 and the decreased expression of COL2A1 and SOX9 induced by IL-1 $\beta$ [98].

Protocatechuic acid, one of polyphenolic compounds extracted from green tea and catechins, possesses analgesic and anti-inflammatory activity. Protocatechuic acid can promote the proliferation of rabbit chondrocytes and maintain cell phenotype through enhancing the synthesis of ECM and the expression of aggrecan, type II collagen, and SOX9 [99]. Psoralen, an effective component isolated from Fructus Psoraleae, dose-dependently enhances the production of GAG and type II collagen and increases the expression of SOX9 [100]. On the other hand, high dose administration of psoralen may induce writhing, lassitude, and hypoactivity. But the significant toxic side effects of psoralen on bone marrow or other organs such as heart, lung, liver, and spleen are not seen [101].

However, among a vast number of chemicals related to cigarette smoking, nicotine is one of the leading candidates 
for causing delayed chondrogenesis. The possible mechanism might be that nicotine increases fetal blood corticosterone, inhibits matrix synthesized by growth plate chondrocytes, and downregulates the expression of IGF-1 signaling in chondrocytes, which promotes longitudinal growth by activating the synthesis of ECM [102]. Additionally, the adverse effects of nicotine have been reported to affect many systems, including neuromuscular, cardiovascular, neurological, immunological, respiratory, and gastrointestinal [103].

\section{Miscellaneous}

Shu-Jing-Huo-Xie-Tang (mainly composed of Cortex Eucommiae), Du-Huo-Ji-Sheng-Tang (Radix Angelicae Pubescentis), and Shao-Yao-Gan-Cao-Tang (Radix Dipsaci) are the most frequency of prescriptions of TCM for diseases of the musculoskeletal system and connective tissue in clinic [104]. Du-Huo-Ji-Sheng-Tang has been demonstrated to show the therapeutic effects on OA in vivo to protect joint tissue through inhibiting the mRNA expression of VEGF and HIF- $1 \alpha$ [105]. Guilu Erxian Jiao (a Chinese traditional formula) is commonly used remedy for knee OA. From the clinical trial, it has been found that Guilu Erxian Jiao can significantly increase muscle strength and decrease joint pain and Lequesne index scores in OA patients after 12 weeks' administration [106].

Tougu Xiaotong capsule formula, a TCM, promotes cell proliferation, reduces cell mortality, and protects cell from inflammatory cytokine, like $\mathrm{TNF} \alpha$, and injury [107]. Yaotongning Capsule (YTNC), a TCM formula, is used for OA treatment in clinic and has a potent activity to prevent OA chondrocytes from degeneration. To improve the quality controllability and safety and achieve maximal therapeutic efficacy, the active fractions of YTNC are reshuttled according to the formulation of YTNC and the concept of combinational chemistry. The results show that the formula can be further simplified according to the reasonable combination of alkaloids, flavonoids, and 50\% of saponins from Glycyrrhiza uralensis, which is an important minister drug in YTNC [108].

Aconitum carmichaeli Debx offers various therapeutic activities, but they are accompanied by acute toxicity due to the aconitine constituents. It can be detoxicated by reducing the aconitine contents. Detoxicated Aconitum carmichaelii Debx shows chondroprotective activity in preventing cartilage degeneration, decreasing the bone density and the Mankin score, and promoting chondrocytes proliferation [109].

HIF- $2 \alpha$ is recently verified to exhibit a crucial role in regulating cartilage destruction by directly inducing expression of catabolic factors, including MMPs, ADAMTS4, iNOS, and prostaglandin-endoperoxide synthase-2 (PTGS2). However, HIF- $2 \alpha$ is regulated by SIRT1. The injection administration of resveratrol can significantly upregulate SIRT1 expression and downregulate the HIF- $2 \alpha$ expression, leading to suppression of iNOS and MMP-13 expression [110]. Our research group shows that Murraya exotica (L.) can dose-dependently downregulate the mRNA and protein expression of $\beta$-catenin and
COX-2, inhibit the levels of TNF $\alpha$ and IL- $\beta$ in synovial fluids, and decrease the chondrocytes apoptosis [111].

\section{Concluding Marks}

This is a systemic review investigating the action mechanisms of TCM and their components regarding activities of antiinflammation, antiapoptosis, antioxidation, anticatabolism, and proliferation in OA. Although the preclinical and clinical trials of TCM are on the initial step, the data collected indicate a promising beneficial effect on OA. Valuable work has been investigated to further explore the clinical merits of TCM (Table 1). They are primarily focusing on inhibition of proinflammatory cytokines activities, suppression of ROSinduced mitochondrial signaling, downregulation of NF- $\kappa \mathrm{B}$, MAPK, and Wnt/ $\beta$-catenin signaling, inhibition of MMPs and ADAMTS activities, and increase of anabolic activity. However, the action mechanisms of TCM in OA have not yet been fully formulated or still under investigation. Under the light of the theory of TCM, the whole combination of different medicines is critically important for its clinical effects. The biological effects of TCM are more than the sum of activities produced by the individual. In addition, because of a vast number of components and the positively or negatively interacting network among these effective compounds, TCM is currently limited by the uncertainty of target specification.

\section{Competing Interests}

The authors declare that they have no conflict of interests.

\section{Authors' Contributions}

Linfu Li, Haiqing Liu, and Weimei Shi contributed equally to this study.

\section{Acknowledgments}

This study was financially supported by the National Science Foundation of China (81360277 and 81660371), the National Science Foundation of Jiangxi Province (20161BAB215219), and Scientific Research Fund of Jiangxi Provincial Education Department (GJJ150959).

\section{References}

[1] R. F. Loeser, S. R. Goldring, C. R. Scanzello, and M. B. Goldring, "Osteoarthritis: a disease of the joint as an organ," Arthritis and Rheumatism, vol. 64, no. 6, pp. 1697-1707, 2012.

[2] M. Blagojevic, C. Jinks, A. Jeffery, and K. P. Jordan, "Risk factors for onset of osteoarthritis of the knee in older adults: a systematic review and meta-analysis," Osteoarthritis and Cartilage, vol. 18, no. 1, pp. 24-33, 2010.

[3] H. Kotlarz, C. L. Gunnarsson, H. Fang, and J. A. Rizzo, "Insurer and out-of-pocket costs of osteoarthritis in the US: evidence from national survey data," Arthritis and Rheumatism, vol. 60, no. 12, pp. 3546-3553, 2009.

[4] F. P. Luyten, P. Tylzanowski, and R. J. Lories, "Wnt signaling and osteoarthritis," Bone, vol. 44, no. 4, pp. 522-527, 2009. 
[5] K. B. Marcu, M. Otero, E. Olivotto, R. M. Borzi, and M. B. Goldring, "NF- $\kappa \mathrm{B}$ signaling: multiple angles to target OA, Current Drug Targets, vol. 11, no. 5, pp. 599-613, 2010.

[6] F. Beier and R. F. Loeser, "Biology and pathology of rho GTPase, PI-3 kinase-Akt, and MAP kinase signaling pathways in chondrocytes," Journal of Cellular Biochemistry, vol. 110, no. 3, pp. 573-580, 2010.

[7] J. Bertrand, C. Cromme, D. Umlauf, S. Frank, and T. Pap, "Molecular mechanisms of cartilage remodelling in osteoarthritis," International Journal of Biochemistry and Cell Biology, vol. 42, no. 10, pp. 1594-1601, 2010.

[8] L. Wu, X. Huang, L. Li, H. Huang, R. Xu, and W. Luyten, "Insights on biology and pathology of HIF- $1 \alpha /-2 \alpha$ TGF $\beta /$ BMP, Wnt $/ \beta$-catenin, and NF- $\kappa \mathrm{B}$ pathways in osteoarthritis," Current Pharmaceutical Design, vol. 18, no. 22, pp. 3293-3312, 2012.

[9] A. Anandacoomarasamy and L. March, "Current evidence for osteoarthritis treatments," Therapeutic Advances in Musculoskeletal Disease, vol. 2, no. 1, pp. 17-28, 2010.

[10] X. Shen and R. Gatti, "The safety and efficacy of intra-articular dual molecular weighted hyaluronic acid in the treatment of knee osteoarthritis: the I.D.E.H.A. study," Orthopedic Reviews, vol. 5, no. 4, p. e33, 2013.

[11] Y. Henrotin, C. Lambert, D. Couchourel, C. Ripoll, and E. Chiotelli, "Nutraceuticals: Do they represent a new era in the management of osteoarthritis? - a narrative review from the lessons taken with five products," Osteoarthritis and Cartilage, vol. 19, no. 1, pp. 1-21, 2011.

[12] D. J. Leong, M. Choudhury, D. M. Hirsh, J. A. Hardin, N. J. Cobelli, and H. B. Sun, "Nutraceuticals: potential for chondroprotection and molecular targeting of osteoarthritis," International Journal of Molecular Sciences, vol. 14, no. 11, pp. 23063-23085, 2013.

[13] L. Wu, H. Liu, L. Li et al., "Mitochondrial pathology in Osteoarthritic chondrocytes," Current Drug Targets, vol. 15, no. 7, pp. 710-719, 2014.

[14] S.-N. Wang, G.-P. Xie, C.-H. Qin et al., "Aucubin prevents interleukin-1 beta induced inflammation and cartilage matrix degradation via inhibition of NF- $\kappa \mathrm{B}$ signaling pathway in rat articular chondrocytes," International Immunopharmacology, vol. 24, no. 2, pp. 408-415, 2015.

[15] Y. J. Chen, K. S. Tsai, D. C. Chan et al., "Honokiol, a low molecular weight natural product, prevents inflammatory response and cartilage matrix degradation in human osteoarthritis chondrocytes," Journal of Orthopaedic Research, vol. 32, no. 4, pp. 573580, 2014.

[16] Q. Zhang, X. Ye, L. Wang et al., "Embryo-fetal development toxicity of honokiol microemulsion intravenously administered to pregnant rats," Regulatory Toxicology and Pharmacology, vol. 74, pp. 117-122, 2016.

[17] Q.-H. Ding, Y. Cheng, W.-P. Chen, H.-M. Zhong, and X.H. Wang, "Celastrol, an inhibitor of heat shock protein $90 \beta$ potently suppresses the expression of matrix metalloproteinases, inducible nitric oxide synthase and cyclooxygenase2 in primary human osteoarthritic chondrocytes," European Journal of Pharmacology, vol. 708, no. 1-3, pp. 1-7, 2013.

[18] X. Ying, X. Chen, S. Cheng, Y. Shen, L. Peng, and H. Xu, "Piperine inhibits IL- $\beta$ induced expression of inflammatory mediators in human osteoarthritis chondrocyte," International Immunopharmacology, vol. 17, no. 2, pp. 293-299, 2013.
[19] C.-C. Cheng, Y.-H. Chen, W.-L. Chang et al., "Phytoestrogen bavachin mediates anti-inflammation targeting $\mathrm{I} \kappa \mathrm{B}$ kinase$\mathrm{I} \kappa \mathrm{B} \alpha-\mathrm{NF}-\kappa \mathrm{B}$ signaling pathway in chondrocytes in vitro," European Journal of Pharmacology, vol. 636, no. 1-3, pp. 181-188, 2010.

[20] Z. Ma, T. Piao, Y. Wang, and J. Liu, "Astragalin inhibits IL- $1 \beta$-induced inflammatory mediators production in human osteoarthritis chondrocyte by inhibiting NF- $\kappa \mathrm{B}$ and MAPK activation," International Immunopharmacology, vol. 25, no. 1, pp. 83-87, 2015.

[21] A. Haseeb, D. Chen, and T. M. Haqqi, "Delphinidin inhibits IL- $1 \beta$-induced activation of NF- $\kappa \mathrm{B}$ by modulating the phosphorylation of IRAK-1 ${ }^{\text {Ser376 }}$ in human articular chondrocytes," Rheumatology, vol. 52, no. 6, pp. 998-1008, 2013.

[22] L.-N. Gao, Q.-S. Feng, X.-F. Zhang, Q.-S. Wang, and Y.-L. Cui, "Tetrandrine suppresses articular inflammatory response by inhibiting pro-inflammatory factors via NF- $\kappa$ B inactivation," Journal of Orthopaedic Research, vol. 34, no. 9, pp. 1557-1568, 2016.

[23] H. Jin, L. Li, D. Zhong, J. Liu, X. Chen, and J. Zheng, "Pulmonary toxicity and metabolic activation of tetrandrine in CD-1 mice," Chemical Research in Toxicology, vol. 24, no. 12, pp. 2142-2152, 2011.

[24] M. Lei, J.-G. Wang, D.-M. Xiao et al., "Resveratrol inhibits interleukin $1 \beta$-mediated inducible nitric oxide synthase expression in articular chondrocytes by activating SIRT1 and thereby suppressing nuclear factor- $\kappa \mathrm{B}$ activity," European Journal of Pharmacology, vol. 674, no. 2-3, pp. 73-79, 2012.

[25] J. Wang, J.-S. Gao, J.-W. Chen, F. Li, and J. Tian, "Effect of resveratrol on cartilage protection and apoptosis inhibition in experimental osteoarthritis of rabbit," Rheumatology International, vol. 32, no. 6, pp. 1541-1548, 2012.

[26] M. Maepa, M. Razwinani, and S. Motaung, "Effects of resveratrol on collagen type II protein in the superficial and middle zone chondrocytes of porcine articular cartilage," Journal of Ethnopharmacology, vol. 178, pp. 25-33, 2016.

[27] S. Ahmed, "Green tea polyphenol epigallocatechin 3-gallate in arthritis: progress and promise," Arthritis Research and Therapy, vol. 12, no. 2, article no. 208, 2010.

[28] G.-S. Huang, C.-Y. Tseng, C.-H. Lee, S.-L. Su, and H.-S. Lee, "Effects of (-)-epigallocatechin-3-gallate on cyclooxygenase 2, $\mathrm{PGE}_{2}$, and IL- 8 expression induced by IL- $1 \beta$ in human synovial fibroblast," Rheumatology International, vol. 30, no. 9, pp. 11971203, 2010.

[29] F. Oliviero, P. Sfriso, A. Scanu, U. Fiocco, P. Spinella, and L. Punzi, "Epigallocatechin-3-gallate reduces inflammation induced by calcium pyrophosphate crystals in vitro," Frontiers in Pharmacology, vol. 4, article 51, 2013.

[30] N. Akhtar and T. M. Haqqi, "Epigallocatechin-3-gallate suppresses the global interleukin-lbeta-induced inflammatory response in human chondrocytes," Arthritis Research and Therapy, vol. 13, no. 3, article no. R93, 2011.

[31] D. J. Leong, M. Choudhury, R. Hanstein et al., "Green tea polyphenol treatment is chondroprotective, anti-inflammatory and palliative in a mouse posttraumatic osteoarthritis model," Arthritis Research \& Therapy, vol. 16, no. 1, article 508, 2014.

[32] S.-C. Liu, H.-P. Lee, C.-Y. Hung, C.-H. Tsai, T.-M. Li, and C.$\mathrm{H}$. Tang, "Berberine attenuates CCN2-induced IL-1 $\beta$ expression and prevents cartilage degradation in a rat model of osteoarthritis," Toxicology and Applied Pharmacology, vol. 289, no. 1, pp. 20-29, 2015. 
[33] C.-M. Wu, T.-M. Li, T.-W. Tan, Y.-C. Fong, and C.-H. Tang, "Berberine reduces the metastasis of chondrosarcoma by modulating the $\alpha \mathrm{v} \beta 3$ integrin and the PKC $\delta$, c-Src, and AP-1 signaling pathways," Evidence-based Complementary and Alternative Medicine, vol. 2013, Article ID 423164, 10 pages, 2013.

[34] J. Bao, B. Huang, L. Zou et al., "Hormetic effect of berberine attenuates the anticancer activity of chemotherapeutic agents," PLoS ONE, vol. 10, no. 9, Article ID e0139298, 2015.

[35] R. F. P. Schelbergen, A. B. Blom, M. H. J. van den Bosch et al., "Alarmins S100A8 and S100A9 elicit a catabolic effect in human osteoarthritic chondrocytes that is dependent on toll-like receptor 4," Arthritis \& Rheumatism, vol. 64, no. 5, pp. 1477-1487, 2012.

[36] H. S. Youn, J. Y. Lee, K. A. Fitzgerald, H. A. Young, S. Akira, and D. H. Hwang, "Specific inhibition of MyD88-independent signaling pathways of TLR3 and TLR4 by resveratrol: molecular targets are TBK1 and RIP1 in TRIF complex," Journal of Immunology, vol. 175, no. 5, pp. 3339-3346, 2005.

[37] L. Liu, H. Gu, H. Liu et al., "Protective effect of resveratrol against IL- $1 \beta$-induced inflammatory response on human osteoarthritic chondrocytes partly via the TLR4/MyD88/NF- $\kappa \mathrm{B}$ signaling pathway: An 'In Vitro Study,' International Journal of Molecular Sciences, vol. 15, no. 4, pp. 6925-6940, 2014.

[38] S.-M. Yu and S.-J. Kim, "Production of reactive oxygen species by withaferin A causes loss of type collagen expression and COX-2 expression through the PI3K/Akt, p38, and JNK pathways in rabbit articular chondrocytes," Experimental Cell Research, vol. 319, no. 18, pp. 2822-2834, 2013.

[39] J.-Y. Na, S. Kim, K. Song et al., "Anti-apoptotic activity of ginsenoside $\mathrm{Rb} 1$ in hydrogen peroxide-treated chondrocytes: stabilization of mitochondria and the inhibition of caspase-3," Journal of Ginseng Research, vol. 36, no. 3, pp. 242-247, 2012.

[40] S. Kim, J.-Y. Na, K.-B. Song et al., "Protective effect of ginsenoside $\mathrm{Rb} 1$ on hydrogen peroxide-induced oxidative stress in rat articular chondrocytes," Journal of Ginseng Research, vol. 36, no. 2, pp. 161-168, 2012.

[41] W. Cheng, D. Wu, Q. Zuo, Z. Wang, and W. Fan, "Ginsenoside $\mathrm{Rbl}$ prevents interleukin-1 beta induced inflammation and apoptosis in human articular chondrocytes," International Orthopaedics, vol. 37, no. 10, pp. 2065-2070, 2013.

[42] Y. Huang, D. Wu, and W. Fan, "Protection of ginsenoside Rg1 on chondrocyte from IL-1 $\beta$-induced mitochondria-activated apoptosis through PI3K/Akt signaling," Molecular and Cellular Biochemistry, vol. 392, no. 1-2, pp. 249-257, 2014.

[43] M. W. So, E.-J. Lee, H. S. Lee et al., "Protective effects of ginsenoside Rg3 on human osteoarthritic chondrocytes," Modern Rheumatology, vol. 23, no. 1, pp. 104-111, 2013.

[44] J. H. Lee, H. Lim, O. Shehzad, Y. S. Kim, and H. P. Kim, "Ginsenosides from Korean red ginseng inhibit matrix metalloproteinase-13 expression in articular chondrocytes and prevent cartilage degradation," European Journal of Pharmacology, vol. 724, no. 1, pp. 145-151, 2014.

[45] X.-H. Zhang, X.-X. Xu, and T. Xu, "Ginsenoside Ro suppresses interleukin- $1 \beta$-induced apoptosis and inflammation in rat chondrocytes by inhibiting NF- $\kappa \mathrm{B}$," Chinese Journal of Natural Medicines, vol. 13, no. 4, pp. 283-289, 2015.

[46] X. Zhang, Y. Zhu, X. Chen et al., "Baicalein ameliorates inflammatory-related apoptotic and catabolic phenotypes in human chondrocytes," International Immunopharmacology, vol. 21, no. 2, pp. 301-308, 2014.

[47] Y. Zhou, S.-Q. Liu, L. Yu et al., "Berberine prevents nitric oxideinduced rat chondrocyte apoptosis and cartilage degeneration in a rat osteoarthritis model via AMPK and p38 MAPK signaling," Apoptosis, vol. 20, no. 9, pp. 1187-1199, 2015.

[48] Y. Zhou, S.-Q. Liu, H. Peng, L. Yu, B. He, and Q. Zhao, "In vivo anti-apoptosis activity of novel berberine-loaded chitosan nanoparticles effectively ameliorates osteoarthritis," International Immunopharmacology, vol. 28, no. 1, pp. 34-43, 2015.

[49] Q. Liang, X.-P. Wang, and T.-S. Chen, "Resveratrol protects rabbit articular chondrocyte against sodium nitroprussideinduced apoptosis via scavenging ROS," Apoptosis, vol. 19, no. 9, pp. 1354-1363, 2014.

[50] H. Jin, Q. Liang, T. Chen, and X. Wang, "Resveratrol protects chondrocytes from apoptosis via altering the ultrastructural and biomechanical properties: an AFM study," PLoS ONE, vol. 9, no. 3, Article ID e91611, 2014.

[51] F. Liu, G. Liu, W. Liang et al., "Duhuo Jisheng decoction treatment inhibits the sodium nitroprussiate-induced apoptosis of chondrocytes through the mitochondrial-dependent signaling pathway," International Journal of Molecular Medicine, vol. 34, no. 6, pp. 1573-1580, 2014.

[52] P. Jia, G. Chen, G. Zhou, Y. Zhong, and R. Li, "Fuyuan Decoction inhibits nitric oxide production via inactivation of nuclear factor- $\kappa$ B in SW1353 chondrosarcoma cells," Journal of Ethnopharmacology, vol. 146, no. 3, pp. 853-858, 2013.

[53] X.-D. Ju, M. Deng, Y.-F. Ao et al., "The protective effect of tetramethylpyrazine on cartilage explants and chondrocytes," Journal of Ethnopharmacology, vol. 132, no. 2, pp. 414-420, 2010.

[54] P. Lin, X. Weng, F. Liu et al., "Bushen Zhuangjin decoction inhibits TM-induced chondrocyte apoptosis mediated by endoplasmic reticulum stress," International Journal of Molecular Medicine, vol. 36, no. 6, pp. 1519-1528, 2015.

[55] L. Wu, H. Liu, L. Li et al., "5,7,3/,4/-Tetramethoxyflavone exhibits chondroprotective activity by targeting $\beta$-catenin signaling in vivo and in vitro," Biochemical and Biophysical Research Communications, vol. 452, no. 3, pp. 682-688, 2014.

[56] J. Yang, H. Liu, L. Li, H. Liu, W. Shi, and L. Wu, "The chondroprotective role of TMF in $\mathrm{PGE}_{2}$-induced apoptosis associating with endoplasmic reticulum stress," Evidence-based Complementary and Alternative Medicine, vol. 2015, Article ID 297423, 7 pages, 2015.

[57] C. Heeschen, J. J. Jang, M. Weis et al., "Nicotine stimulates angiogenesis and promotes tumor growth and atherosclerosis," Nature Medicine, vol. 7, no. 7, pp. 833-839, 2001.

[58] X. Zheng, C. Xia, Z. Chen et al., "Requirement of the phosphatidylinositol 3-kinase/Akt signaling pathway for the effect of nicotine on interleukin-1beta-induced chondrocyte apoptosis in a rat model of osteoarthritis," Biochemical and Biophysical Research Communications, vol. 423, no. 3, pp. 606-612, 2012.

[59] Y. Gao, S. Liu, J. Huang et al., "The ECM-cell interaction of cartilage extracellular matrix on chondrocytes," BioMed Research International, vol. 2014, Article ID 648459, 8 pages, 2014.

[60] F. Zhu, L. Yin, L. Ji et al., "Suppressive effect of Sanmiao formula on experimental gouty arthritis by inhibiting cartilage matrix degradation: An In Vivo and In Vitro Study," International Immunopharmacology, vol. 30, pp. 36-42, 2016.

[61] D.-Q. Wu, H.-M. Zhong, Q.-H. Ding, and L. Ba, "Protective effects of biochanin A on articular cartilage: in vitro and in vivo studies," BMC Complementary and Alternative Medicine, vol. 14, no. 1, article 444, 2014.

[62] S. H. Alavizadeh and H. Hosseinzadeh, "Bioactivity assessment and toxicity of crocin: a comprehensive review, Food and Chemical Toxicology, vol. 64, pp. 65-80, 2014. 
[63] Q. Ding, H. Zhong, Y. Qi et al., "Anti-arthritic effects of crocin in interleukin- $\beta$-treated articular chondrocytes and cartilage in a rabbit osteoarthritic model," Inflammation Research, vol. 62, no. 1, pp. 17-25, 2013.

[64] P.-W. Yuan, H.-C. Yu, H.-Z. Zhou, C. Zhu, Q. Qu, and D.Y. Liu, "Preventive administration of juanbi capsules for knee osteoarthritis: effects on serum MMP-2 and MMP-9 levels and cartilage repair," Journal of Traditional Chinese Medicine, vol. 31, no. 4, pp. 334-337, 2011.

[65] X.-D. Ju, M. Deng, Y.-F. Ao et al., "Protective effect of sinomenine on cartilage degradation and chondrocytes apoptosis," Yakugaku Zasshi, vol. 130, no. 8, pp. 1053-1060, 2010.

[66] F. Wang, L. Wu, L. Li, and S. Chen, "Monotropein exerts protective effects against IL- $1 \beta$-induced apoptosis and catabolic responses on osteoarthritis chondrocytes," International Immunopharmacology, vol. 23, no. 2, pp. 575-580, 2014.

[67] P.-F. Hu, W.-P. Chen, J.-L. Tang, J.-P. Bao, and L.-D. Wu, "Protective effects of berberine in an experimental rat osteoarthritis model," Phytotherapy Research, vol. 25, no. 6, pp. 878-885, 2011.

[68] H. Zhao, T. Zhang, C. Xia et al., "Berberine ameliorates cartilage degeneration in interleukin-1 $\beta$-stimulated rat chondrocytes and in a rat model of osteoarthritis via Akt signalling," Journal of Cellular and Molecular Medicine, vol. 18, no. 2, pp. 283-292, 2014.

[69] J.-H. Kim, J.-E. Huh, Y.-H. Baek, J.-D. Lee, D.-Y. Choi, and D.-S. Park, "Effect of Phellodendron amurense in protecting human osteoarthritic cartilage and chondrocytes," Journal of Ethnopharmacology, vol. 134, no. 2, pp. 234-242, 2011.

[70] L. Zhao, J. Ye, G.-T. Wu, X.-J. Peng, P.-F. Xia, and Y. Ren, “Gentiopicroside prevents interleukin-1 beta induced inflammation response in rat articular chondrocyte," Journal of Ethnopharmacology, vol. 172, article no. 9593, pp. 100-107, 2015.

[71] W.-P. Chen, Y. Xiong, P.-F. Hu, J.-P. Bao, and L.-D. Wu, "Baicalein inhibits MMPs expression via a MAPK-dependent mechanism in chondrocytes," Cellular Physiology and Biochemistry, vol. 36, no. 1, pp. 325-333, 2015.

[72] Q.-Q. Liang, D.-F. Ding, Z.-J. Xi et al., "Protective Effect of ligustrazine on lumbar intervertebral disc degeneration of rats induced by prolonged upright posture," Evidence-based Complementary and Alternative Medicine, vol. 2014, Article ID 508461, 9 pages, 2014.

[73] W.-P. Chen, Y. Xiong, Y.-X. Shi, P.-F. Hu, J.-P. Bao, and L.-D. $\mathrm{Wu}$, "Astaxanthin reduces matrix metalloproteinase expression in human chondrocytes," International Immunopharmacology, vol. 19, no. 1, pp. 174-177, 2014.

[74] Y.-J. Jeong, J.-M. Shin, Y.-S. Bae et al., "Melittin has a chondroprotective effect by inhibiting MMP-1 and MMP-8 expressions via blocking NF- $\kappa \mathrm{B}$ and AP-1 signaling pathway in chondrocytes," International Immunopharmacology, vol. 25, no. 2, pp. 400-405, 2016.

[75] J.-W. Jeong, H. H. Lee, E.-O. Choi et al., “Schisandrae Fructus inhibits IL- $1 \beta$-induced matrix metalloproteinases and inflammatory mediators production in SW1353 human chondrocytes by suppressing NF- $\kappa$ B and MAPK activation," Drug Development Research, vol. 76, no. 8, pp. 474-483, 2015.

[76] W.-P. Chen, P.-F. Hu, J.-P. Bao, and L.-D. Wu, "Morin exerts antiosteoarthritic properties: an in vitro and in vivo study," Experimental Biology and Medicine, vol. 237, no. 4, pp. 380-386, 2012.

[77] H. Lu, J. Jiang, G. Xie, W. Liu, and G. Yan, "Effects of an aqueous extract of Eucommia on articular cartilage in a rat model of osteoarthritis of the knee," Experimental and Therapeutic Medicine, vol. 6, no. 3, pp. 684-688, 2013.

[78] G.-P. Xie, N. Jiang, S.-N. Wang et al., "Eucommia ulmoides Oliv. bark aqueous extract inhibits osteoarthritis in a rat model of osteoarthritis," Journal of Ethnopharmacology, vol. 162, pp. 148154, 2015.

[79] D. Li, T. Yuan, X. Zhang et al., "Icariin: a potential promoting compound for cartilage tissue engineering," Osteoarthritis and Cartilage, vol. 20, no. 12, pp. 1647-1656, 2012.

[80] W. Zhang, R. Li, S. Wang, X. Zhou, and Y. Zhong, "Study of molecular mechanisms of Fuyuan capsule, icariin and arasaponin $\mathrm{R}_{1}$ in treatment of osteoarthritis," Zhongguo Zhongyao Zazhi, vol. 36, no. 15, pp. 2113-2117, 2011.

[81] L. Zeng, W. Wang, X.-F. Rong et al., "Chondroprotective effects and multi-target mechanisms of Icariin in IL-1 beta-induced human SW 1353 chondrosarcoma cells and a rat osteoarthritis model," International Immunopharmacology, vol. 18, no. 1, pp. 175-181, 2014.

[82] W. Wang, L. Zeng, Z.-M. Wang, S. Zhang, X.-F. Rong, and R.-H. Li, "Ginsenoside Rb1 inhibits matrix metalloproteinase 13 through down-regulating Notch signaling pathway in osteoarthritis," Experimental Biology and Medicine, vol. 240, no. 12, pp. 1614-1621, 2015.

[83] D. Zhang, B. Huang, C. Xiong, and Z. Yue, "Pinocembrin inhibits matrix metalloproteinase expression in chondrocytes," IUBMB Life, vol. 67, no. 1, pp. 36-41, 2015.

[84] M. P. Chen, S. H. Yang, C. H. Chou et al., "The chondroprotective effects of ferulic acid on hydrogen peroxide-stimulated chondrocytes: inhibition of hydrogen peroxide-induced proinflammatory cytokines and metalloproteinase gene expression at the mRNA level," Inflammation Research, vol. 59, no. 8, pp. 587-595, 2010.

[85] X. Zhou, W. Li, L. Jiang et al., “Tetrandrine inhibits the Wnt/ $\beta$ catenin signalling pathway and alleviates osteoarthritis: An In Vitro and In Vivo Study," Evidence-based Complementary and Alternative Medicine, vol. 2013, Article ID 809579, 8 pages, 2013.

[86] X. Lu, R. Zhang, F. Fu, J. Shen, H. Nian, and T. Wu, "Simultaneous determination of fangchinoline and tetrandrine in QiFang-Xi-Bi-granules by RP-HPLC," Journal of Chromatographic Science, vol. 53, no. 8, pp. 1328-1332, 2015.

[87] C.-Y. Huang, L.-F. Hung, C.-C. T. Liang, and L.-J. Ho, "COX2 and iNOS are critical in advanced glycation end productactivated chondrocytes in vitro," European Journal of Clinical Investigation, vol. 39, no. 5, pp. 417-428, 2009.

[88] F.-C. Liu, L.-F. Hung, W.-L. Wu et al., "Chondroprotective effects and mechanisms of resveratrol in advanced glycation end products-stimulated chondrocytes," Arthritis Research o Therapy, vol. 12, no. 5, article R167, 2010.

[89] M. Shakibaei, A. Mobasheri, and C. Buhrmann, "Curcumin synergizes with resveratrol to stimulate the MAPK signaling pathway in human articular chondrocytes in vitro," Genes and Nutrition, vol. 6, no. 2, pp. 171-179, 2011.

[90] K. V. Peddada, K. V. Peddada, S. K. Shukla, A. Mishra, and V. Verma, "Role of curcumin in common musculoskeletal disorders: a review of current laboratory, translational, and clinical data," Orthopaedic Surgery, vol. 7, no. 3, pp. 222-231, 2015.

[91] V. Natarajan, B. Madhan, and M. L. Tiku, "Intra-articular injections of polyphenols protect articular cartilage from inflammation-induced degradation: suggesting a potential role in cartilage therapeutics," PLoS ONE, vol. 10, no. 6, Article ID e0127165, 2015 . 
[92] M.-N. Horcajada, C. Sanchez, F. Membrez Scalfo et al., "Oleuropein or rutin consumption decreases the spontaneous development of osteoarthritis in the Hartley guinea pig," Osteoarthritis and Cartilage, vol. 23, no. 1, pp. 94-102, 2015.

[93] W. Wu, X. Xu, Y. Dai, and L. Xia, “Therapeutic effect of the saponin fraction from Clematis chinensis osbeck roots on osteoarthritis induced by monosodium iodoacetate through protecting articular cartilage," Phytotherapy Research, vol. 24, no. 4, pp. 538-546, 2010.

[94] X. Weng, P. Lin, F. Liu et al., "Achyranthes bidentata polysaccharides activate the $\mathrm{Wnt} / \beta$-catenin signaling pathway to promote chondrocyte proliferation," International Journal of Molecular Medicine, vol. 34, no. 4, pp. 1045-1050, 2014.

[95] X. Li, J. Chen, W. Liang et al., "Bushen Zhuangjin Decoction promotes chondrocyte proliferation by stimulating cell cycle progression," Experimental and Therapeutic Medicine, vol. 9, no. 3, pp. 839-844, 2015.

[96] G. Wu, W. Chen, H. Fan et al., "Duhuo Jisheng Decoction promotes chondrocyte proliferation through accelerated G1/S transition in osteoarthritis," International Journal of Molecular Medicine, vol. 32, no. 5, pp. 1001-1010, 2013.

[97] H. Li, X. Li, G. Liu et al., "Bauhinia championi (Benth.) Benth. polysaccharides upregulate $\mathrm{Wnt} / \beta$-catenin signaling in chondrocytes," International Journal of Molecular Medicine, vol. 32, no. 6, pp. 1329-1336, 2013.

[98] Y. Zhang, R. Li, Y. Zhong, S. Zhang, L. Zhou, and S. Shang, "Fuyuan decoction enhances SOX9 and COL2A1 expression and Smad2/3 phosphorylation in IL- $1 \beta$-activated chondrocytes," Evidence-Based Complementary and Alternative Medicine, vol. 2015, Article ID 821947, 9 pages, 2015.

[99] L. Luo, Q. Wei, L. Liu et al., "Protocatechuic acid benefits proliferation and phenotypic maintenance of rabbit articular chondrocytes: An In Vitro Study," Experimental and Therapeutic Medicine, vol. 9, no. 5, pp. 1865-1870, 2015.

[100] K. Xu, X. Pan, Y. Sun, W. Xu, L. Njunge, and L. Yang, "Psoralen activates cartilaginous cellular functions of rat chondrocytes in vitro," Pharmaceutical Biology, vol. 53, no. 7, pp. 1010-1015, 2015.

[101] H. Lu, L. Zhang, D. Liu, P. Tang, and F. Song, "Isolation and purification of psoralen and isopsoralen and their efficacy and safety in the treatment of osteosarcoma in nude rats," African Health Sciences, vol. 14, no. 3, pp. 641-647, 2014.

[102] Y. Deng, H. Cao, F. Cu et al., "Nicotine-induced retardation of chondrogenesis through down-regulation of IGF-1 signaling pathway to inhibit matrix synthesis of growth plate chondrocytes in fetal rats," Toxicology and Applied Pharmacology, vol. 269, no. 1, pp. 25-33, 2013.

[103] P. N. Lee and M. W. Fariss, "A systematic review of possible serious adverse health effects of nicotine replacement therapy," Archives of Toxicology, pp. 1-30, 2016.

[104] P.-R. Yang, H.-F. Liang, Y.-H. Chu, P.-C. Chen, and Y.-Y. Lin, "Frequencies and prescription patterns of traditional Chinese medicine use among elderly patients in Taiwan: a populationbased study," Journal of Ethnopharmacology, vol. 169, pp. 328334, 2015.

[105] C.-W. Chen, J. Sun, Y.-M. Li, P.-A. Shen, and Y.-Q. Chen, "Action mechanisms of Du-Huo-Ji-Sheng-Tang on cartilage degradation in a rabbit model of osteoarthritis," Evidence-based Complementary and Alternative Medicine, vol. 2011, Article ID 571479, 7 pages, 2011.

[106] C.-C. Tsai, Y.-Y. Chou, Y.-M. Chen, Y.-J. Tang, H.-C. Ho, and D.Y. Chen, "Effect of the herbal drug Guilu Erxian Jiao on muscle strength, articular pain, and disability in elderly men with knee osteoarthritis," Evidence-Based Complementary and Alternative Medicine, vol. 2014, Article ID 297458, 9 pages, 2014.

[107] N. Liao, Y. Huang, J. Ye et al., "Protective effects of Tougu Xiaotong capsule on tumor necrosis factor- $\alpha$-injured UMR-106 cells," Experimental and Therapeutic Medicine, vol. 10, no. 5, pp. 1908-1914, 2015.

[108] L.-G. Zhang, X.-W. Ouyang, T.-T. Wu, L.-J. Ni, and W.-Z. Shi, "Quantitative evaluation of in vitro effects and interactions of active fractions in a Chinese medicinal formula (Yaotongning Capsule) on rat chondrocytes," Journal of Ethnopharmacology, vol. 155, no. 3, pp. 1424-1432, 2014.

[109] P. Tong, S. Xu, G. Cao et al., "Chondroprotective activity of a detoxicated traditional Chinese medicine (Fuzi) of Aconitum carmichaeli Debx against severe-stage osteoarthritis model induced by mono-iodoacetate," Journal of Ethnopharmacology, vol. 151, no. 1, pp. 740-744, 2014.

[110] W. Li, L. Cai, Y. Zhang, L. Cui, and G. Shen, "Intra-articular resveratrol injection prevents osteoarthritis progression in a mouse model by activating SIRT1 and thereby silencing HIF2a," Journal of Orthopaedic Research, vol. 33, no. 7, pp. 1061-1070, 2015.

[111] L. Wu, H. Liu, R. Zhang et al., "Chondroprotective activity of Murraya exotica through inhibiting $\beta$-catenin signaling pathway," Evidence-based Complementary and Alternative Medicine, vol. 2013, Article ID 752150, 8 pages, 2013. 


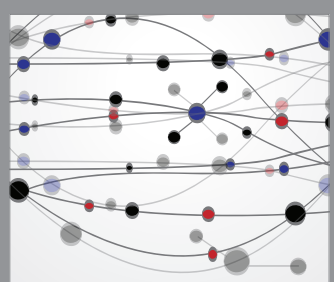

The Scientific World Journal
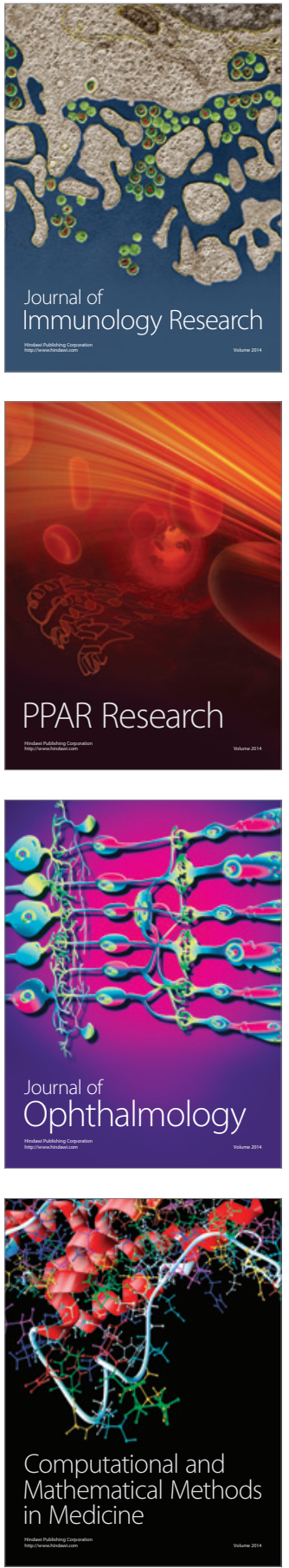

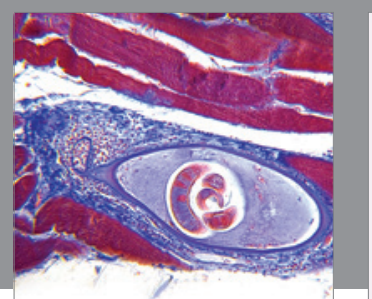

Gastroenterology Research and Practice
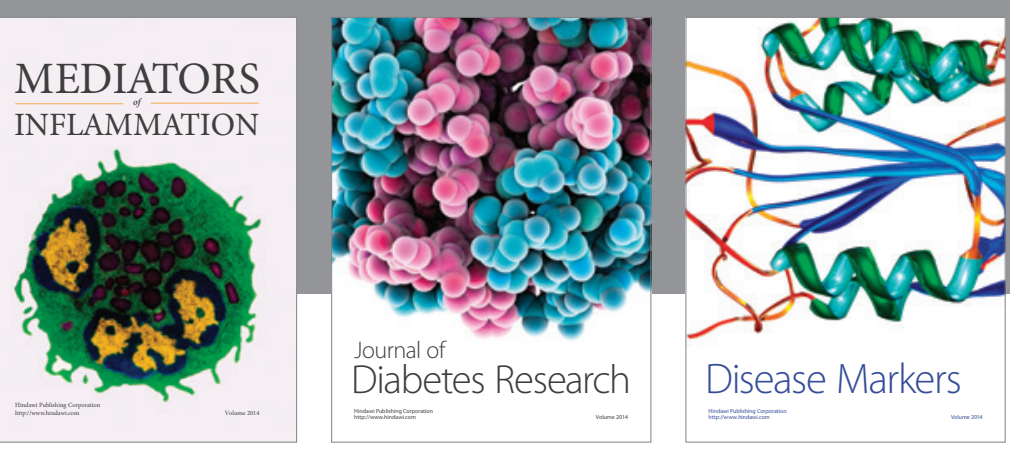

Disease Markers

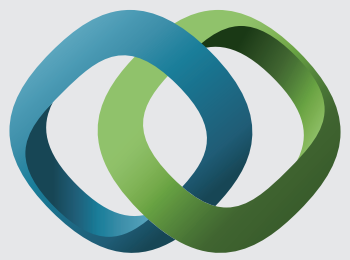

\section{Hindawi}

Submit your manuscripts at

https://www.hindawi.com
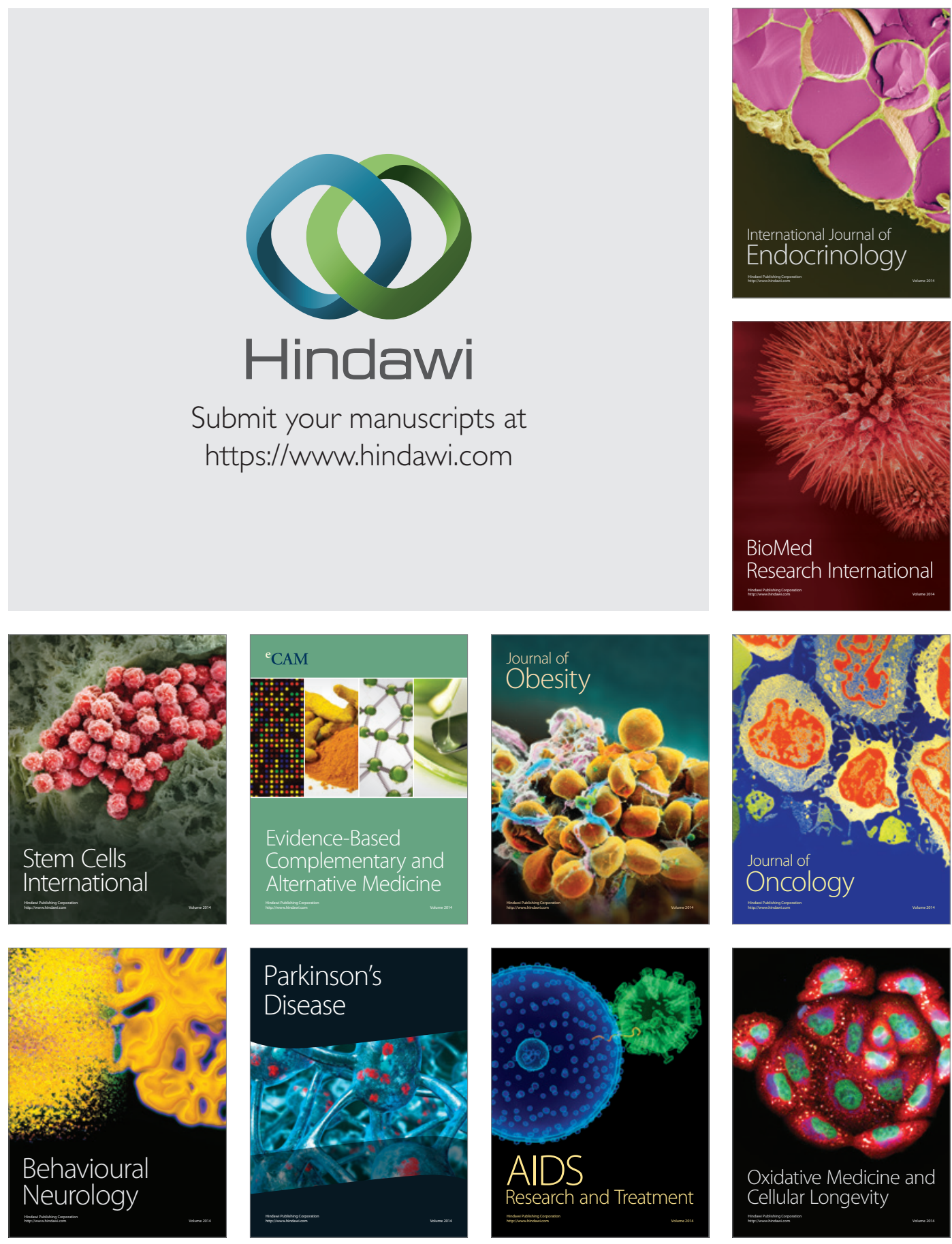